\title{
RISK PRIORITIZATION OF ENVIRONMENTAL ASPECTS AND OCCUPATIONAL HEALTH SAFETY IN TEXTILE INDUSTRIES OF GAZIPUR INDUSTRIAL AREA, BANGLADESH
}

\author{
Md. Iftakharul Muhib \\ Assistant Professor \\ General Education Department \\ City University, Dhaka, Bangladesh \\ E-mail: muhibiftakhar@gmail.com \\ Md. Shawkut Ali Khan \\ Professor \& Head \\ Department of Mechanical Engineering \\ City University, Dhaka, Bangladesh \\ E-mail: shawkutali8@gmail.com \\ Rifat Akter \\ Post Graduate Student \\ Department of Microbiology \\ Jagannath University, Dhaka, Bangladesh \\ E-mail: rifatakter5@gmail.com
}

\begin{abstract}
Though textile industries are the backbone of country's economy; it possesses the environmental and occupational health safety risks also. After the Rana Plaza tragedy, the environmental and occupational health safety regulations become started to come spotlight. However, still there found anomalies that threaten environmental and occupational health safety. So to get an overview of present risk levels in different industries, the aim of this study was set to evaluate the risk prioritization for both environmental aspects and occupational health safety. A total of 10 textile industries were selected and a direct field survey along with an interview from workers to top management was done to conduct the study. Risk assessment results for environmental aspects showed that water pollution shows a moderate risk rating value while air quality, soil quality, noise, waste generation, hazardous material handling, and miscellaneous use showed low risks. Again calculated results for occupational health safety showed that necessary action is recommended to avoid future risk in all-hazard categories of different types except for electrical hazards. Controlling measures were suggested in accordance with the risk priority. Thus it is high time to explore and evaluate the risk level and take necessary action on the resultant priority basis.
\end{abstract}

Keywords: Environmental Aspects, Occupational Health Safety, Risk, RPN, Textile Industry.

\section{INTRODUCTION}

Textile industries play a pivotal role in the rapid economic growth of Bangladesh (World Bank, 2012). The contribution of the textile sector to the country's total export revenue is more than $80 \%$ that is 28 billion USD/year and the projected revenue is 50 billion USD by the tear of 2021 (Hossain et al., 2018). However, this great success comes with environmental deterioration along with occupational health safety risks. Different types of environmental aspects like water discharge, air quality, soil 
quality, noise, waste generation, energy consumed, a resource used, hazardous materials storage, and miscellaneous issues were considered to be major sources of environmental impacts in the Gazipur industrial area. Heavy metals like $\mathrm{Cr}, \mathrm{Ni}, \mathrm{Zn}, \mathrm{Hg}, \mathrm{Ob}, \mathrm{Cu}, \mathrm{Cd}$, As, etc. are released from the textile industries that pollute the river, soil, and consequently the food chain when this polluted water is used for irrigation purpose to cultivate the seasonal crops (Islam et al., 2013). Several occupational health hazards are found in the textile industries of Bangladesh. Some of them were so drastic that international textile market trade was likely to fall on its edge. One of the worst examples is the Rana plaza accidental disaster in 2013 that killed over 1100 workers and the devastating fire of Tajreen garments in 2012 killed over 100 workers (Khan, 2017). So risk leveling of environmental aspects and occupational health safety along with their remedial measures is very important to avoid the disaster. However, enforcement of these remedial in perspective of risk rating is very much challenging. The reasons for enforcement hindrance are insufficient resources (knowledge, human resource, and funds), the unwillingness of industry owners, economic corruption, political imbalance, and inadequate coordination among the concerned agencies. Since there is no sufficient data for risk evaluation of environmental aspects and occupational health safety hazards, this study could act as a baseline survey for further rigorous study regarding the environmental and occupational compliance sector of Bangladesh.

\section{METHODOLOGY}

This study was conducted to confine the current status of environmental aspects and occupational health safety scenarios in the scale of risk evaluation to develop a research hypothesis. A total of 10 different textile industries in the Gazipur area were selected to conduct the survey. This survey was designed to collect information about the environmental aspects and occupational health safety practices in the selected industries directly from the field survey as well as interviewed from the worker to top management. All the collected data were processed and analyzed chronologically. One of the real constraints of the investigation was their privacy maintenance. Taking pictures and gathering information inside the factory premises were very much challenging since the authorities didn't want to disclose their in site scenario publicly. Therefore none of the names of studied industries are disclosed in this manuscript.

\section{Data Responders}

A total of 490 respondents were selected from 10 industries where 100 data responders (10 from each industry) from management and 390 respondents (39 from each industry) from the worker level. In this analysis, about 98\% (38 out of 39 respondents) of management body know very well about environmental and occupational compliance while only $15 \%$ (59 out of 390 respondents) workers know about these compliances in very primary level and rest of 85\% (331 out of 390 respondents) workers know nothing about this matter.

\section{Risk Evaluation for Environmental Aspects}

The identification of environmental aspects and impacts is important to the selection of environmental safeguards and work methods for operational activities. Risk evaluation of environmental aspects depicts the magnitude of potential consequence (level of environmental impacts) and the likelihood (level of probability of occurrence) (PERSEUS, 2012).

\section{Risk $(\mathbf{R})=$ Likelihood $(\mathrm{L}) \times$ Consequence $(\mathrm{C})$}

This $\mathrm{C} \times \mathrm{L}$ matrix displays the qualitative or semi-quantitative rating of consequence and likelihood that specific data will occur to generate a risk score and risk rating. Risk rating data shows the level of risk in absence of a controlled environment and is arrived at after measuring the livelihood and the consequence of an event occurring (Endeavour Technical Limited, 2015). 


\section{Risk Evaluation for Occupational Health Safety}

The Failure Mode and Impact Analysis (FMEA) method are followed for occupational risk safety analysis (Yari, 2017; Wang et al., 2018). This method enhances the safety and reliability level by providing early detection and elimination of failures (SAE International, 2020). In this way, risk prioritization is done by taking into the multiplication of severity, probability, and detectability that is known as Risk Priority Number or RPN (Yang \& Wang, 2015; Zhang \& Chu, 2011).

\section{RPN $=$ Severity $\times$ Probability $\times$ Detectability}

Here, severity is the consequence of the failure; occurrence is the probability or the frequency of failure occurring, and detection is the probability of the failure being detected before the impact of the effect is realized. If the RPN result crosses 100, necessary action is recommended. The maximum value of RPN is 1000 (Kumar et al., 2014).

\section{Risk Rating for Environmental Aspects}

\section{RESULTS AND DISCUSSION}

Environmental aspects can be attributed to the activity that interferes with the natural environmental condition called the impact that is the resultant of an aspect (Susanto \& Mulyono, 2018).

Table 1. Risk Assessment Level of Environmental Aspects

\begin{tabular}{|c|c|c|c|c|c|c|c|c|c|}
\hline \multirow{3}{*}{$\begin{array}{c}\text { Environmental } \\
\text { Aspects }\end{array}$} & \multirow{3}{*}{$\begin{array}{l}\text { Potential } \\
\text { Impacts }\end{array}$} & \multirow{3}{*}{$\begin{array}{c}\text { Identified } \\
\text { Risk }\end{array}$} & \multicolumn{4}{|c|}{ Risk Evaluation } & \multirow{2}{*}{\multicolumn{2}{|c|}{ Risk Rating }} & \multirow[t]{3}{*}{ Control Measures/Mitigation } \\
\hline & & & \multicolumn{2}{|c|}{ Likelihood } & \multicolumn{2}{|c|}{ Consequence } & & & \\
\hline & & & Condition & Score & Condition & Score & Score & $\begin{array}{l}\begin{array}{l}\text { Level } \\
\text { of Risk }\end{array} \\
\end{array}$ & \\
\hline $\begin{array}{c}\text { Water } \\
\text { Discharge }\end{array}$ & $\begin{array}{c}\text { Water } \\
\text { Pollution }\end{array}$ & $\begin{array}{l}\text { >Disposal } \\
\text { of } \\
\text { chemical. } \\
\text { >Effluent } \\
\text { from ETP. } \\
\text { >Disposal } \\
\text { of used oil } \\
\text { for } \\
\text { machine } \\
\text { maintenan } \\
\text { ces. } \\
\text { >Sewage } \\
\text { discharge }\end{array}$ & Possible & 3 & Moderate & 2 & 6 & $\begin{array}{l}\text { Modera } \\
\text { te Risk }\end{array}$ & $\begin{array}{l}>\text { Chemical store should be } \\
\text { maintained properly. } \\
>\text { Optimization of Effluent } \\
\text { Treatment Plant (ETP) operation is } \\
\text { highly recommended. }\end{array}$ \\
\hline Air Quality & $\begin{array}{c}\text { Air } \\
\text { Pollution } \\
\text { and Dust } \\
\text { Emission }\end{array}$ & $\begin{array}{l}\text { Dust and } \\
\text { other } \\
\text { exhaust } \\
\text { atmospher } \\
\text { ic } \\
\text { emission } \\
\text { i.e. } \\
\text { emission } \\
\text { of } \mathrm{SO}_{2} \text {, } \\
\mathrm{NO}_{\mathrm{x}}, \mathrm{CO} \text {, } \\
\mathrm{SPM} \text {, etc. } \\
\text { occur due } \\
\text { to the } \\
\text { stack gas } \\
\text { of } \\
\text { generator } \\
\text { and } \\
\text { vehicular } \\
\text { movement } \\
\text { in and } \\
\text { outside of } \\
\text { the factory } \\
\text { area. }\end{array}$ & Possible & 3 & Minor & 1 & 3 & $\begin{array}{l}\text { Low } \\
\text { Risk }\end{array}$ & $\begin{array}{l}>\text { Factory chimney should be } \\
\text { functional } 24 / 7 \text {. } \\
>\text { Water spraying can be done to } \\
\text { reduce dust pollution on the nearby } \\
\text { road. } \\
>\text { Ensuring vehicles, generators, } \\
\text { compressors are well maintained. } \\
>\text { Proper ventilation system should } \\
\text { be designed. } \\
>\text { Use of PPE like a mask, apron } \\
\text { should be used mandatorily }\end{array}$ \\
\hline Soil Quality & $\begin{array}{c}\text { Soil } \\
\text { Pollution }\end{array}$ & $\begin{array}{l}>\text { Leakage } \\
\text { of oil from } \\
\text { vehicles }\end{array}$ & Unlikely & 2 & Moderate & 2 & 4 & $\begin{array}{l}\text { Low } \\
\text { Risk }\end{array}$ & $\begin{array}{l}\text { >Proper solid waste management } \\
\text { should be followed }\end{array}$ \\
\hline
\end{tabular}




\begin{tabular}{|c|c|c|c|c|c|c|c|c|c|}
\hline & $\begin{array}{c}\text { and } \\
\text { Erosion }\end{array}$ & $\begin{array}{l}\text { and other } \\
\text { maintenan } \\
\text { ce works } \\
>\text { Mainten } \\
\text { ance work } \\
\text { that } \\
\text { excavates } \\
\text { soil. } \\
\text { >Chemica } \\
1 \text { spillage }\end{array}$ & & & & & & & \\
\hline Noise & $\begin{array}{c}\text { Noise } \\
\text { Pollution }\end{array}$ & $\begin{array}{l}>\text { Noise is } \\
\text { generated } \\
\text { from } \\
\text { vehicle } \\
\text { movement } \\
\text {, operation } \\
\text { of the } \\
\text { generator, } \\
\text { compress } \\
\text { or, and } \\
\text { other } \\
\text { heavy } \\
\text { vibrating } \\
\text { types of } \\
\text { machinery }\end{array}$ & Possible & 3 & Minor & 1 & 3 & $\begin{array}{l}\text { Low } \\
\text { Risk }\end{array}$ & $\begin{array}{l}\text { More use of PPE like earplug/ear } \\
\text { muffs in noisy workplace }\end{array}$ \\
\hline $\begin{array}{c}\text { Waste } \\
\text { Generation }\end{array}$ & $\begin{array}{l}\text { Surroundi } \\
\text { ng } \\
\text { Environm } \\
\text { ental } \\
\text { Pollution }\end{array}$ & $\begin{array}{l}\text { >Impact } \\
\text { of } \\
\text { disposal } \\
\text { waste } \\
\text { >Hazardo } \\
\text { us } \\
\text { chemical/ } \\
\text { biomedica } \\
1 \text { waste. } \\
\text { >Non- } \\
\text { persistent } \\
\text { material } \\
\text { e.g. paper, } \\
\text { cardboard, } \\
\text { etc. } \\
\text { >Persisten } \\
\text { t material } \\
\text { e.g. } \\
\text { plastic, } \\
\text { scrap, etc. } \\
\text { >E-waste. } \\
\text { >Kitchen } \\
\text { waste. }\end{array}$ & Possible & 3 & Minor & 1 & 3 & $\begin{array}{l}\text { Low } \\
\text { Risk }\end{array}$ & $\begin{array}{l}\text { > Introducing 3R (Reduce, Reuse, } \\
\text { Recycle) waste management. } \\
\text { > Medical waste generated by the } \\
\text { factory should be handed over to the } \\
\text { local hospital. }\end{array}$ \\
\hline Energy Used & $\begin{array}{l}\text { Power } \\
\text { Consumpt } \\
\text { ion }\end{array}$ & $\begin{array}{l}\text { >Chemica } \\
\text { l/oil } \\
\text { spillage in } \\
\text { the } \\
\text { generator } \\
\text { room. } \\
>\mathrm{Pb}, \mathrm{Cd} \text {, } \\
\mathrm{Hg} \\
\text { pollution } \\
\text { from scrap } \\
\text { battery } \\
\text { and solar } \\
\text { panel }\end{array}$ & Unlikely & 2 & Minor & 1 & 2 & $\begin{array}{l}\text { No- } \\
\text { Risk }\end{array}$ & $\begin{array}{l}\text { > Handling of chemicals and oil } \\
\text { should be done properly. } \\
>\text { Older battery and solar panel } \\
\text { should be disposed of properly }\end{array}$ \\
\hline Resources Used & $\begin{array}{l}\text { Infrastruct } \\
\text { ure } \\
\text { Developm } \\
\text { ent }\end{array}$ & $\begin{array}{l}\text { >Land. } \\
>\text { Building } \\
\text { > types of } \\
\text { machinery } \\
\text { > Tools. } \\
>\text { Trees } \\
\text { etc. }\end{array}$ & Unlikely & 2 & Minor & 1 & 2 & $\begin{array}{l}\text { No- } \\
\text { Risk }\end{array}$ & $\begin{array}{l}\text { >Land management and building } \\
\text { design should be done properly. }\end{array}$ \\
\hline $\begin{array}{c}\text { Ecological } \\
\text { View (Flora \& } \\
\text { Fauna) }\end{array}$ & $\begin{array}{l}\text { Loss of } \\
\text { Habitat of } \\
\text { some } \\
\text { Flora and }\end{array}$ & $\begin{array}{l}\text { >Unplann } \\
\text { ed } \\
\text { constructi }\end{array}$ & Unlikely & 2 & Minor & 1 & 2 & $\begin{array}{l}\text { No- } \\
\text { Risk }\end{array}$ & $\begin{array}{l}\text { >Maintain maximum vegetation. } \\
\text { > Implementation of agroforestry } \\
\text { techniques well adapted to the site. }\end{array}$ \\
\hline
\end{tabular}




\begin{tabular}{|c|c|c|c|c|c|c|c|c|c|}
\hline & $\begin{array}{c}\text { Fauna and } \\
\text { Biodiversi } \\
\text { ty } \\
\text { Reduction }\end{array}$ & $\begin{array}{l}\text { on and } \\
\text { design } \\
\text { >Inapprop } \\
\text { riate } \\
\text { control of } \\
\text { weeds }\end{array}$ & & & & & & & \\
\hline $\begin{array}{l}\text { Hazardous } \\
\text { Materials } \\
\text { Storage and } \\
\text { Handling }\end{array}$ & $\begin{array}{l}\text { Occurrenc } \\
\text { e of } \\
\text { accidents }\end{array}$ & $\begin{array}{l}\text { Accidenta } \\
1 \text { spillage } \\
\text { and fire } \\
\text { occur due } \\
\text { to } \\
\text { personal } \\
\text { error or } \\
\text { natural } \\
\text { events. }\end{array}$ & Unlikely & 2 & Moderate & 2 & 4 & $\begin{array}{l}\text { Low } \\
\text { Risk }\end{array}$ & $\begin{array}{l}\text { >Proper inspection of maintenance } \\
\text { and storage of hazardous goods } \\
\text { should be monitored regularly. }\end{array}$ \\
\hline $\begin{array}{l}\text { Miscellaneous } \\
\text { Issues }\end{array}$ & $\begin{array}{l}\text { Others } \\
\text { Incidents }\end{array}$ & $\begin{array}{l}\text { > Imbalan } \\
\text { ce of } \\
\text { political } \\
\text { condition. } \\
\text { > Worker } \\
\text { imbalance } \\
\text { > Producti } \\
\text { on loss. } \\
>\text { Visual } \\
\text { appearanc } \\
\text { e. }\end{array}$ & Unlikely & 2 & Moderate & 2 & 4 & $\begin{array}{l}\text { Low } \\
\text { Risk }\end{array}$ & $\begin{array}{l}>\text { Management should be aware of } \\
\text { workers legal demand }\end{array}$ \\
\hline
\end{tabular}

There is a significant amount of water, energy and other resources are consumed by the studied industries. Based on an environmental point of view, a list of environmental; aspects, their risk rating along controlling measures are organized in Table 1. A moderate risk rating was found for water pollution impacts. Though there was ETP in most of the studied industries, there showed some anomalies like ETP did not run 24 hours, lack of ETP servicing hindered the ETP efficiency, personal error, maintenance, and chemical cost and last but not least is the lack of ETP performance optimization. Other environmental aspects like soil quality, air quality, noise, waste generation, hazardous material handling, and miscellaneous issues though were found to be possessed low risk, they could be upgraded to more negative risk levels in the near future if a proper controlling measure is not taken immediately. However environmental aspects like energy used, a resource used, and ecological view in the industrial area were found to be relatively at the safe level from risk. All the control measures are given accordingly.

\section{Risk Rating for Occupational Health and Safety}

Spinning, weaving, dyeing, printing, and garments sections were studied in the 10 industries. Hazards occurred in these industries are classified into 7 different categories- physical hazard, fire hazard, electrical hazard, chemical hazard, ergonomic hazard, psychosocial hazard, and biological hazard. These categories were subdivided into different hazard types. The consequences of these hazards were rated with an RPN number to recommend whether necessary action would be required or not (Table 2). Remedial measures are also listed in the same table. Risk prioritization is found for most of the hazard types. The highest number of RPN (RPN=384) was found for hazard type of dyes from the chemical hazard category that causes water pollution in the nearby water bodies though ETP was found in almost all the surveyed industries. The cause of this RPN number either the lack of proper operational optimization or lack of proper handling of dyes materials. These dyes material are hazardous to workers' health. For example, dyes can cause skin and eye irritation, lung inflammation, and other respiratory problems (Bansal \& Yadhav, 2016). 
Table 2. Risk Leveling of Occupational Health Hazards

\begin{tabular}{|c|c|c|c|c|c|}
\hline $\begin{array}{c}\text { Hazard } \\
\text { Category }\end{array}$ & Hazard Type & Consequences & $\begin{array}{c}\text { RPN } \\
(\text { Probability } \times \text { Detection } \times \text { Severity })\end{array}$ & $\begin{array}{l}\text { Necessity } \\
\text { of Action }\end{array}$ & $\begin{array}{l}\text { Remedial } \\
\text { Measures }\end{array}$ \\
\hline \multirow[t]{5}{*}{$\begin{array}{l}\text { Physical } \\
\text { Hazard }\end{array}$} & Noise & Hearing loss & $10 \times 6 \times 6=360$ & $\begin{array}{c}\text { Action } \\
\text { Required }\end{array}$ & $\begin{array}{l}>\text { Proper } \\
\text { servicing of } \\
\text { the heavy } \\
\text { types of } \\
\text { machinery. } \\
>\text { Use of } \\
\text { soundproof } \\
\text { materials } \\
\text { and earplug }\end{array}$ \\
\hline & Dust & $\begin{array}{l}\text { Respiratory } \\
\text { Disease }\end{array}$ & $8 \times 7 \times 6=336$ & $\begin{array}{c}\text { Action } \\
\text { Required }\end{array}$ & $\begin{array}{l}\text { >Proper } \\
\text { housekeepi } \\
\text { ng } \\
\text { >Use of } \\
\text { PPE widely }\end{array}$ \\
\hline & Light & Eye stress & $3 \times 3 \times 3=27$ & $\begin{array}{c}\text { Not } \\
\text { Required }\end{array}$ & $\begin{array}{l}\text { >Optimized } \\
\text { light set up }\end{array}$ \\
\hline & Temperature & $\begin{array}{c}\text { High } \\
\text { temperature } \\
\text { create } \\
\text { dehydration } \\
\text { and blood } \\
\text { pressure } \\
\text { anomalies } \\
\end{array}$ & $8 \times 6 \times 6=288$ & $\begin{array}{l}\text { Action } \\
\text { Required }\end{array}$ & $\begin{array}{l}>\text { Pigh } \\
\text { temperature } \\
\text { in the } \\
\text { working } \\
\text { place should } \\
\text { be } \\
\text { controlled }\end{array}$ \\
\hline & $\begin{array}{l}\text { Improper } \\
\text { Ventilation }\end{array}$ & $\begin{array}{l}\text { Lung function } \\
\text { Change }\end{array}$ & $4 \times 3 \times 3=36$ & $\begin{array}{c}\text { Not } \\
\text { Required }\end{array}$ & $\begin{array}{l}\text { >Proper } \\
\text { ventilation } \\
\text { system } \\
\text { should be } \\
\text { installed }\end{array}$ \\
\hline \multirow[t]{2}{*}{ Fire Hazard } & Welding & Spark ignition & $3 \times 2 \times 3=18$ & $\begin{array}{c}\text { Not } \\
\text { Required }\end{array}$ & $\begin{array}{l}>\text { Restrict } \\
\text { the welding } \\
\text { area for } \\
\text { movement }\end{array}$ \\
\hline & Smoking & $\begin{array}{l}\text { Common } \\
\text { accidental } \\
\text { source }\end{array}$ & $5 \times 4 \times 5=100$ & $\begin{array}{c}\text { Action } \\
\text { Required }\end{array}$ & $\begin{array}{l}>\text { Smoking } \\
\text { should be } \\
\text { banned in } \\
\text { the working } \\
\text { place. } \\
>\text { Safety } \\
\text { sign should } \\
\text { be } \\
\text { maintained }\end{array}$ \\
\hline \multirow[t]{3}{*}{$\begin{array}{l}\text { Electrical } \\
\text { Hazard }\end{array}$} & $\begin{array}{l}\text { Improper } \\
\text { Earthing }\end{array}$ & Trip occurs & $3 \times 2 \times 3=18$ & $\begin{array}{c}\text { Not } \\
\text { Required }\end{array}$ & $\begin{array}{l}>\text { Should } \\
\text { check } \\
\text { earthing } \\
\text { connection } \\
\text { properly }\end{array}$ \\
\hline & Short Circuit & $\begin{array}{l}\text { A common } \\
\text { source of the } \\
\text { fire accident }\end{array}$ & $5 \times 3 \times 6=90$ & $\begin{array}{c}\text { Not } \\
\text { Required }\end{array}$ & $\begin{array}{l}>\text { Air Circuit } \\
\text { breaker and } \\
\text { motor } \\
\text { circuit } \\
\text { breaker } \\
\text { should be } \\
\text { installed. }\end{array}$ \\
\hline & $\begin{array}{l}\text { Improper } \\
\text { Isolation }\end{array}$ & Electric shock & $3 \times 3 \times 4=36$ & $\begin{array}{c}\text { Not } \\
\text { Required }\end{array}$ & $\begin{array}{l}\text { All circuits } \\
\text { should be }\end{array}$ \\
\hline
\end{tabular}




\begin{tabular}{|c|c|c|c|c|c|}
\hline & & & & & $\begin{array}{l}\text { installed } \\
\text { properly. }\end{array}$ \\
\hline & $\begin{array}{c}\text { Motor Rotation } \\
\text { in High } \\
\text { Voltage }\end{array}$ & $\begin{array}{l}\text { Problems } \\
\text { occur in } \\
\text { machine } \\
\text { performance }\end{array}$ & $3 \times 3 \times 3=27$ & $\begin{array}{c}\text { Not } \\
\text { Required }\end{array}$ & $\begin{array}{l}\text { > Individual } \\
\text { circuit } \\
\text { breaker } \\
\text { should be } \\
\text { installed. }\end{array}$ \\
\hline & Old Wiring & $\begin{array}{l}\text { Anomalies in } \\
\text { current flow }\end{array}$ & $6 \times 4 \times 4=96$ & $\begin{array}{c}\text { Not } \\
\text { Required }\end{array}$ & $\begin{array}{l}\text { Check for } \\
\text { old wiring } \\
\text { to avoid } \\
\text { accidents. }\end{array}$ \\
\hline & $\begin{array}{l}\text { Power Input- } \\
\text { Output } \\
\text { Imbalance }\end{array}$ & $\begin{array}{c}\text { Power shut } \\
\text { down }\end{array}$ & $3 \times 4 \times 2=24$ & $\begin{array}{c}\text { Not } \\
\text { Required }\end{array}$ & $\begin{array}{l}>\text { Wiring } \\
\text { should be } \\
\text { done } \\
\text { according to } \\
\text { the } \\
\text { standards. }\end{array}$ \\
\hline \multirow[t]{3}{*}{$\begin{array}{c}\text { Chemical } \\
\text { Hazard }\end{array}$} & Dyes & $\begin{array}{c}\text { Skin and lung } \\
\text { disease }\end{array}$ & $8 \times 6 \times 8=384$ & $\begin{array}{c}\text { Action } \\
\text { Required }\end{array}$ & $\begin{array}{l}>\text { ETP must } \\
\text { be installed } \\
\text { with } \\
\text { optimizatio } \\
\mathrm{n}\end{array}$ \\
\hline & $\begin{array}{l}\text { Different } \\
\text { Types of } \\
\text { Chemical }\end{array}$ & $\begin{array}{l}\text { Skin and lung } \\
\text { disease }\end{array}$ & $7 \times 6 \times 6=252$ & $\begin{array}{c}\text { Action } \\
\text { Required }\end{array}$ & $\begin{array}{l}\text { >Proper } \\
\text { handling } \\
\text { and storage } \\
\text { should be } \\
\text { followed }\end{array}$ \\
\hline & $\begin{array}{l}\text { Used oil and } \\
\text { grease }\end{array}$ & $\begin{array}{c}\text { Skin and lung } \\
\text { disease }\end{array}$ & $6 \times 5 \times 5=150$ & $\begin{array}{c}\text { Action } \\
\text { Required }\end{array}$ & $\begin{array}{l}\text { >Proper } \\
\text { handling } \\
\text { and storage } \\
\text { should be } \\
\text { followed }\end{array}$ \\
\hline \multirow[t]{5}{*}{$\begin{array}{c}\text { Ergonomic } \\
\text { Hazard }\end{array}$} & $\begin{array}{l}\text { Uncomfortable } \\
\text { Work station }\end{array}$ & $\begin{array}{l}\text { Leg and hand } \\
\text { pain }\end{array}$ & $4 \times 4 \times 3=48$ & $\begin{array}{c}\text { Not } \\
\text { Required }\end{array}$ & $\begin{array}{l}>\text { Should } \\
\text { give } \\
\text { importance } \\
\text { to } \\
\text { ergonomics }\end{array}$ \\
\hline & $\begin{array}{c}\text { Repetitive } \\
\text { Strain Injuries }\end{array}$ & $\begin{array}{c}\text { Wrist, neck, } \\
\text { shoulder, knee } \\
\text { pain }\end{array}$ & $6 \times 5 \times 6=180$ & $\begin{array}{c}\text { Action } \\
\text { Required }\end{array}$ & $\begin{array}{l}\text { >Proper } \\
\text { working } \\
\text { procedure } \\
\text { should be } \\
\text { followed. }\end{array}$ \\
\hline & $\begin{array}{l}\text { Improper } \\
\text { Handling of } \\
\text { Material }\end{array}$ & $\begin{array}{l}\text { Wrist, neck } \\
\text { pain }\end{array}$ & $4 \times 4 \times 4=64$ & $\begin{array}{c}\text { Not } \\
\text { Required }\end{array}$ & $\begin{array}{l}\text { >Proper } \\
\text { handling } \\
\text { and storage } \\
\text { should be } \\
\text { followed }\end{array}$ \\
\hline & $\begin{array}{c}\text { Poorly } \\
\text { Designed Work } \\
\text { Practice }\end{array}$ & $\begin{array}{l}\text { Hand pain, } \\
\text { back pain }\end{array}$ & $2 \times 3 \times 4=24$ & $\begin{array}{c}\text { Not } \\
\text { Required }\end{array}$ & $\begin{array}{l}>\text { Proper } \\
\text { working } \\
\text { procedure } \\
\text { should be } \\
\text { followed }\end{array}$ \\
\hline & $\begin{array}{l}\text { Long Time } \\
\text { sitting }\end{array}$ & $\begin{array}{l}\text { Headache, } \\
\text { dizziness, } \\
\text { nausea }\end{array}$ & $5 \times 5 \times 6=150$ & $\begin{array}{c}\text { Action } \\
\text { Required }\end{array}$ & $\begin{array}{l}\text { >Should } \\
\text { take a break } \\
\text { at least } 5 \\
\text { minutes } \\
\text { after 1-hour } \\
\text { documental } \\
\text { work }\end{array}$ \\
\hline
\end{tabular}




\begin{tabular}{|c|c|c|c|c|c|}
\hline & $\begin{array}{l}\text { Heavy Weight } \\
\text { Lifting }\end{array}$ & Back pain & $3 \times 3 \times 3=27$ & $\begin{array}{c}\text { Not } \\
\text { Required }\end{array}$ & $\begin{array}{l}>\text { Should not } \\
\text { uplift the } \\
\text { heavyweigh } \\
t \text { alone. }\end{array}$ \\
\hline & $\begin{array}{l}\text { Unsafe } \quad \text { Work } \\
\text { Place }\end{array}$ & $\begin{array}{l}\text { Accidents may } \\
\text { occur }\end{array}$ & $3 \times 3 \times 4=36$ & $\begin{array}{c}\text { Not } \\
\text { Required }\end{array}$ & $\begin{array}{l}\text { Working } \\
\text { place should } \\
\text { be designed } \\
\text { with safety } \\
\text { measures. }\end{array}$ \\
\hline \multirow[t]{5}{*}{$\begin{array}{c}\text { Psychosocial } \\
\text { Hazard }\end{array}$} & $\begin{array}{c}\text { Not Interested } \\
\text { to Work } \\
\text { willingly due to } \\
\text { lack of } \\
\text { Motivation }\end{array}$ & $\begin{array}{l}\text { Physiological } \\
\text { problem }\end{array}$ & $7 \times 5 \times 6=210$ & $\begin{array}{c}\text { Action } \\
\text { Required }\end{array}$ & $\begin{array}{l}\text { >Working } \\
\text { department } \\
\text { should be } \\
\text { favorable. } \\
\text { > Holiday, } \\
\text { bonus, } \\
\text { salary } \\
\text { should be } \\
\text { given } \\
\text { properly }\end{array}$ \\
\hline & $\begin{array}{c}\text { Production } \\
\text { Target }\end{array}$ & Stress & $6 \times 5 \times 5=150$ & $\begin{array}{c}\text { Action } \\
\text { Required }\end{array}$ & $\begin{array}{l}\text { The } \\
\text { working } \\
\text { department } \\
\text { should be } \\
\text { favorable. }\end{array}$ \\
\hline & $\begin{array}{l}\text { Increased } \\
\text { Workload }\end{array}$ & $\begin{array}{l}\text { Stress, heart } \\
\text { rate changes }\end{array}$ & $7 \times 6 \times 6=252$ & $\begin{array}{c}\text { Action } \\
\text { Required }\end{array}$ & $\begin{array}{l}\text { The } \\
\text { workload } \\
\text { should be } \\
\text { distributed } \\
\text { evenly. }\end{array}$ \\
\hline & Night Shift & $\begin{array}{c}\text { Headache, } \\
\text { dizziness, } \\
\text { nausea }\end{array}$ & $5 \times 4 \times 5=100$ & $\begin{array}{c}\text { Action } \\
\text { Required }\end{array}$ & $\begin{array}{l}\text { The } \\
\text { working } \\
\text { department } \\
\text { should be } \\
\text { favorable. }\end{array}$ \\
\hline & Over Time & Stress, tiered & $5 \times 5 \times 5=125$ & $\begin{array}{c}\text { Action } \\
\text { Required }\end{array}$ & $\begin{array}{l}\text { The } \\
\text { working } \\
\text { department } \\
\text { should be } \\
\text { favorable. }\end{array}$ \\
\hline $\begin{array}{l}\text { Biological } \\
\text { Hazard }\end{array}$ & $\begin{array}{c}\text { Contagious } \\
\text { Diseases }\end{array}$ & $\begin{array}{c}\text { Fever, pox, } \\
\text { TB, Covid-19 }\end{array}$ & $5 \times 5 \times 5=125$ & $\begin{array}{c}\text { Action } \\
\text { Required }\end{array}$ & $\begin{array}{l}\text { Always } \\
\text { should be } \\
\text { cautious } \\
\text { about if a } \\
\text { person feels } \\
\text { ill or } \\
\text { affected by } \\
\text { any other } \\
\text { disease } \\
\text { especially } \\
\text { contagious } \\
\text { disease. }\end{array}$ \\
\hline
\end{tabular}

The least RPN number (RPN=18) was found for improper earthing of electrical hazard. This means that earthing was found to be done properly in all the studied industries. According to the RPN data, the descending order of listed hazards are leveled in the following order- physical hazard: noise>dust>temperature>improper ventilation>light; fire hazard: smoking>welding; an electrical hazard: old wiring > short circuit>improper isolation $>$ high voltage motor rotation>power input-output imbalance; a chemical hazard: dyes>chemicals>used oil and grease; an ergonomic hazard: repetitive 
strain injury>long time sitting>Improper material handling>Uncomfortable work station>unsafe work place>heavy weight lifting >poorly designed work practice; a psychosocial hazard: increased work load>willingness to work>production target>over time>night shift. This type of risk prioritization is also done by Kumar et al. (2014); Liu et al. (2012) and Chang (2016). The resultants o these RPN values make the risk understandable and recommend taking necessary action whether it is necessary or not to minimize it to a tolerable level. Infection of COVID-19 also seems to be hazardous (Shuvro \& Talukdar, 2020).

\section{CONCLUSION}

Textile industries in the Gazipur area are confronted with a number of significant environmental and occupational health safety challenges. However, none of these challenges are unfeasible to overcome. The resultant risk leveling data for both environmental and occupational health safety scenarios would be of great use to policymakers, urban authorities, planners, researchers, and stalk holders of the industries for exploring towards sustainable development of the country.

\section{ACKNOWLEDGEMENT}

The authors are grateful to the studied industry's top management for giving permission to conduct the survey. The authors have no conflict of interest regarding this study. No funds, grants, or other supports were received. Authors Contribution: Md. Itakharul Muhib: Conceptualization, methodology, investigation, writing original draft; Md. Shawkut Ali Khan: Validation, supervision; Rifat Akter: Writing, review, and editing.

\section{REFERENCES}

Bansal, M., \& Yadav, R. K. (2016). Occupational Health Hazards and Awareness of Occupational Safety Among Workers Of Textile Dyeing Industries In Jaipur, India. OCCUPATIONAL HEALTH, 2(2), 30-38.

Chang, K. H. (2016). Generalized multi-attribute failure mode analysis. Neurocomputing, 175, 90100.

Endeavour Technical Limited. (2015). ISO 14001: 2015. Control of Environmental Aspects \& Impacts. Retrieved from https://www.iso-9001-checklist.co.uk/download/ISO-14001-2015environmental-aspects-and-impacts-procedure-sample.pdf

Hossain, L., Sarker, S. K., \& Khan, M. S. (2018). Evaluation of Present and Future Wastewater Impacts of Textile Dyeing Industries in Bangladesh. Environmental Development, 26, 23-33.

Islam, M. R., Jahiruddin, M., Islam, M. R., \& Alim, M. A. (2013). Consumption of unsafe foods: Evidence from heavy metal, mineral and trace element contamination. The National Food Policy Capacity Strengthening Programme (NFPCSP) sponsored project, Dept. of Soil Science, BAU, Mymensingh. Retrieved from http://fpmu.gov.bd/agridrupal/content/consumption-unsafe-foods-heavy-metal-mineral-andtrace-element-contamination

Khan, M. Z. H. (2017). Environmental Management System of Textile Industry in Bangladesh: Constraints and Remediation. International Jour of Environment, Ecology, Family and Urban Studies, 7(2), 1-12.

Kumar, P. M., Mugundhan, K., \& Visagavel, K. (2014). Occupational Health and Safety in Textile Industry. International Journal of Research in Engineering and Technology, 3(11), 168-172. 
Liu, H. C., Liu, L., Liu, N., \& Mao, L. X. (2012). Risk evaluation in failure mode and effects analysis with extended VIKOR method under fuzzy environment. Expert Systems with Application, 39(17), 12926-12934

PERSEUS. (2012). AMP Tool Box, Key Activity 4. Qualitative Risk Analysis. Retrieved from http://www.perseus-net.eu/site/content.php?artid=2204.

Shuvro, R. A., \& Talukder, M. M. (2020). Impact of "COVID-19" on the Life of Destitute People: A Soft-Measurement in Bangladesh. Bangladesh Journal of Multidisciplinary Scientific Research, 2(2). https://doi.org/10.46281/bjmsr.v2i2.723

Susanto, A. \& Mulyono, N. B. (2018). Risk Assessment Method for Identification of Environmental Aspects and Impacts at Ore Processing Industry in Indonesia. Journal of Ecological Engineering, 19(2), 72-80.

SAE International. (2020). Recommended Failure Modes and Effects Analysis (FMEA) Practices for Non-Automobile Applications. Retrieved from https://www.sae.org/standards/content/arp5580

Wang, W., Liu, X., Qin, Y., \& Fu, Y. (2018). A risk evaluation and prioritization method for FMEA with prospect theory and Choquet integral. Safety Science, 110, 152-163.

World Bank. (2012). Overview. Vol. 1 of Bangladesh-Towards Accelerated, Inclusive and Sustainable Growth: Opportunities and Challenges; World Bank: Washington, DC, USA.

Yang, Z., \& Wang, J. (2015). Use of fuzzy risk assessment in FMEA of offshore engineering systems. Ocean Engineering, 95, 195-204.

Yari, S. (2017). Assessment of potential risk by the failure mode and effects analysis in an air conditioning equipment manufacturing company. Jour of Safety Promotion and Injury Prevention, 5(2).

Zhang, Z., \& Chu, X. (2011). Risk prioritization in failure mode and effects analysis under uncertainty. Expert Systems with Applications. 38(1), 206-214.

\section{Copyrights}

Copyright for this article is retained by the author(s), with first publication rights granted to the journal. This is an open-access article distributed under the terms and conditions of the Creative Commons Attribution license (http://creativecommons.org/licenses/by/4.0/) 